\title{
Problemáticas ambientales; pretexto para potenciar la argumentación científica en población infantil.
}

\section{Problemas ambientais; pretexto para aumentar a argumentação científica em crianças.}

\author{
D. Alejandro Valderrama danielfisicop@gmail.com \\ Grupo de investigación Waira; Ambiente, Comunidad y Desarrollo Grupo de Astrofísica y Cosmología, \\ Licenciado en Ciencias Naturales y Educación Ambiental \\ Universidad Pedagógica y Tecnológica de Colombia. \\ Yamile Pedraza-Jiménez, yamile.pedraza@uptc.edu.co \\ Grupo de Investigación MICRAM, Doctora en Educación, docente de práctica pedagógica, Universidad \\ Pedagógica y Tecnológica de Colombia
}

Resumen: Se presenta una experiencia de aula fundamentada pedagógicamente en los cambios y exigencias ejercidas sobre la educación en ciencias naturales, por el contexto de la pandemia del SARS-CoV-2 y otras situaciones problemáticas que derivan de las relaciones entre el ser humano y el entorno natural. Se concretan dentro de las mismas la necesidad de fortalecer los procesos argumentativos en un grupo de estudiantes de quinto año de educación básica primaria, en una institución educativa de la ciudad de Tunja, Boyacá en Colombia, sobre los que se realiza un análisis del discurso centrado en niveles de argumentación medidos a lo largo de varias sesiones de clase. Se reconocen algunos avances en la incorporación de esta habilidad como objetivo educativo de diferentes áreas del conocimiento y se describe a su vez las potencialidades didácticas que la enseñanza contextual de las ciencias naturales, gestada mediante el uso de problemáticas ambientales, articuladas con los procesos de interpretación de las mismas, fundamentación conceptual y análisis multidimensional, deriva en procesos de discusión y confrontación de saberes que fortalecen la habilidad argumentativa permitiendo un proceso de enseñanza-aprendizaje de las ciencias naturales desde el protagonismo de los estudiantes. Se incentiva un ejercicio responsable de su ciudadanía y toma de decisiones frente al ambiente, desde una visión de la ética del menor daño y la responsabilidad social, económica y política que los ciudadanos poseen frente al equilibrio ambiental.

Palabras-clave: Enseñanza de las ciencias; argumentación; Educación primaria

Resumo: A experiência de sala de aula é apresentada pedagogicamente com base nas mudanças e demandas exercidas na educação em ciências naturais, devido ao contexto da pandemia do SARS-CoV-2 e outras situações problemáticas que derivam das relações entre o ser humano e o ambiente natural. Neles se especifica a necessidade de fortalecer os processos argumentativos em um grupo de alunos do quinto ano do ensino fundamental, de uma instituição de ensino da cidade de Tunja, Boyacá, na Colômbia, na qual se realiza uma análise de discurso focada em os níveis de argumentação medidos ao longo de várias sessões de aula. Reconhecem-se alguns avanços na incorporação desta habilidade como objetivo educativo de diferentes áreas do conhecimento e descrevem-se as potencialidades didáticas do ensino contextual das ciências naturais, 
gestado através da utilização de problemas ambientais, articulados com os processos de interpretação das mesmas. A fundamentação conceitual e análise multidimensional, deriva de processos de discussão e confronto de saberes que fortalecem a capacidade argumentativa permitindo um processo ensino-aprendizagem das ciências naturais a partir do protagonismo dos alunos e autonomia em seu processo de formação.São estimulados a um exercício responsável de sua cidadania e tomada de decisões em relação ao ambiente, a partir de uma visão da ética da redução de danos e da responsabilidade social, econômica e política no equilíbrio ambiental que possuem.

Palavras-chave: Ensino de ciências; argumentação; Educação primária

\section{INTRODUCCION}

La pandemia Covid-19 develó diversas situaciones en los países latinoamericanos que entre otras cosas incluyen la deficiencia de los sistemas de salud y las desigualdades en acceso a los recursos, tecnológicos, logísticos y económicos.

Estas desigualdades afectan de forma directa los procesos administrativos y sociales de la educación (ANDERETE SCHWAL, 2020; CABRERA; PÉREZ; SANTANA, 2020) a partir de esto, la pandemia se constituye como detonante de innovación del quehacer pedagógico y didáctico, ya que dichas brechas que se generan en torno al acceso a la educación, son la génesis de cuestionamientos sobre al rol del docente, algunos de ellos referenciados por autores como González et al., (2020) en términos de la profundidad de la actividad docente, la capacidad y profesionalismo de los educadores en la adaptación a las circunstancias, así como en el uso de nuevas estrategias que permeen las necesidades contextuales y territoriales de sus estudiantes, además de la pertinencia de los contenidos y conocimientos que se construían o compartían en el aula de clases (TARTAVULEA et al., 2020) y la adaptación de modelos evaluativos, que integren los retos de la realidad mundial en el proceso de enseñanza y aprendizaje.

A partir de lo anterior se hace necesario involucrar una serie de recursos que, si bien deben estar sujetos al contexto y realidad socioeconómica de los estudiantes, deben pensarse como posibilitadores de procesos educativos fundamentados en la equidad y la igualdad de derechos de los mismos independientemente de sus aspectos etnográficos y sociodemográficos.

En esa evolución del pensamiento didáctico y particularmente en la enseñanza de las ciencias naturales, toman importancia algunos propósitos generados en algunas 
circunstancias problemáticas diferentes, pero que se validan en una emergencia como la que actualmente se afronta y que es producto de unas relaciones particulares que desarrolla el ser humano con su entorno natural, mediadas a su vez por aspectos culturales, cuyo principal sustento son los diferentes tipos de enseñanza.

Una de estas circunstancias son las problemáticas ambientales, que generalmente están relacionadas con el conflicto de intereses entre los sistemas sociales, económicos y naturales, con grandes afectaciones en este último y un desequilibrio consecuente en el ecosistema, que amenaza al igual que la pandemia, algunas formas de vida en la tierra (ALMUNA, 2013) y que impacta varios ejes contextuales entre ellos el territorio y el desarrollo mismo de la educación.

A partir de lo anterior la educación se convierte en el escenario propicio que posibilita el desarrollo de una serie de competencias, con fundamentos conceptuales y experienciales para asumir posturas razonables, consientes y contextuales en torno a las situaciones particulares que ofrece el territorio y la contemporaneidad histórica en la que el individuo se encuentra inmerso (HERRERA, 2016), sin dejar de lado la necesidad intrínseca de ofrecer bagajes conceptuales, científicos y culturales que le permitan al sujeto reconocer, contrastar, refutar y respetar las cosmovisiones, particularidades y posturas de otros territorios, comunidades e individuos, ya que son precisamente esas habilidades las que incentivan en las sociedades contemporáneas la construcción de nuevos conocimientos que viabilicen nuevas transformaciones (PRESTI, 2006) y den soluciones a las diferentes problemáticas a las que se enfrenta la humanidad.

La consecución de estas habilidades básicas constituidas como retos actuales de la enseñanza trascienden hacia la comprensión de las dinámicas científicas; por lo que para abordar las problemáticas ambientales, se requiere un bagaje de conocimientos particulares desde los aspectos biológicos y ecológicos del entorno natural, pero también las dinámicas físicas y químicas que se involucran en la generación del llamado equilibrio ecosistémico, pues desde la comprensión científica de los fenómenos, se vislumbrarán las problemáticas y se podrán proponer alternativas de relaciones con los mismos. Desde el estudio de las relaciones ecosistémicas, con la cotidianidad cultural en la que se desarrolla el ser humano, se podrá potenciar la capacidad de analizar nuestra manera de habitar el mundo desde una ética del menor daño (PEDRAZA- 
JIMÉNEZ, 2020). Es prioritario para la educación establecer responsabilidades frente al entorno y el territorio que enlazan la necesidad de crear una identidad y soberanía con el mismo. La perspectiva ambiental en los diferentes niveles de formación, se puede lograr al introducir en los diferentes currículos, aspectos que permitan el análisis, reflexión y acción entorno a las relaciones multidimensionales que se desarrollan entre las visiones sociales, políticas, económicas, éticas y culturales con los ecosistemas (PEDRAZAJIMÉNEZ, 2020).

Se pretende entonces a través de estrategias didácticas, generar espacios para la reflexión, de manera que surjan posturas críticas y argumentativas frente al conocimiento y la utilidad del mismo, provocando que los educandos entren en la capacidad de asumir su conocimiento desde un proceso de transformación, desde sí y desde el otro, que se va construyendo con la constante toma de decisiones en los diferentes niveles de vida. (BOTERO; TOBÓN; RAMÍREZ, 2015; MARTÍNEZ PÉREZ; VILLAMIZAR FÚQUENE, 2014; MERCHÁN, 2011).

Todo lo anterior constituye un punto de análisis para el proceso de enseñanza de las ciencias naturales, representado en una experiencia particular que reflejó algunas de las preocupaciones entorno a la educación científica en el contexto de la educación primaria o fundamental que ya habían manifestado en otros escenarios autores como Vazquez; Manassero Mas, (2008) y que están relacionadas con las actitudes negativas e inapropiadas de los estudiantes hacia la ciencia, que incluyen una falta de interés en la misma o una visión monótona y abstracta, que se representa en la baja participación de los estudiantes en las actividades de la clase, o en los cortos análisis que sobre los fenómenos naturales realizan los mismos, esto se vio acrecentado con la situación de pandemia, en la que no sólo se afectaron las emociones de los niños, sino que a la clase entraron en juego unas variables como la lejanía que representa el tener al docente y a los demás niños como una foto de pantalla, o la interacción con un fenómeno desde lo imaginativo y poco vivencial.

De este modo durante la transición de la educación presencial a la educación mediada por las herramientas TIC, se evidencia en los estudiantes de grado 5 de educación básica primaria de una institución educativa colombiana, una baja participación que se limitaba además a un manejo descriptivo y emotivo de las respuestas. 
Los niños asumían posturas frente a su realidad que carecían de un sustento teórico o de elementos que permitieran reconocer una postura argumentada o fundamentado entorno a sus saberes e incluso entorno a las inquietudes que tenían, producto del desconocimiento de un determinado fenómeno biológico o natural. De igual forma, se reconoce entonces que los conocimientos son asumidos únicamente desde la vivencia como experiencia, mas que desde generar una serie de relaciones de de análisis entorno a la importancia que en la vida practica y ordinaria representa profundizar en el conocimiento.

Como se expuso anteriormente, este panorama es preocupante, ya que se aumenta la brecha en la toma de decisiones, frente a situaciones que afrontan a diario los niños y en el futuro cercano, limitando así el ejercicio de construcción política y democrática, pero también el desarrollo científico y tecnológico de los países que depende exclusivamente de las habilidades de análisis, interpretación, cuestionamiento, y argumentación entorno a fenómenos particulares (ACEVEDO, 2005). Desde una comprensión colectiva, estas dificultades son proporcionales al análisis de desigualdad latente en nuestro continente y específicamente de países como Colombia (BARRIOS; ADAM, 2016) que se hizo más visible con la pandemia y que exige de la educación un actuar contundente que solvente y transforme esa realidad.

Sin embargo es necesario también reconocer que en Colombia se ha consolidado una serie de propuestas entorno al desarrollo de la argumentación en todos los niveles educativos y desde diferentes campos del conocimiento, específicamente para el nivel de la básica primaria se han planteado desde la comprensión de la lengua materna(ARIAS; SAPONARA, 2016; HERNÁNDEZ; SERNA; REYNA, 2020), desde la matemática, sobresale la mayor parte de propuestas (ARNAL-BAILERA; GASCA, 2018; CERVANTES-BARRAZA; VALBUENA; PATERNINA, 2019) desde las ciencias sociales; (CIFUENTES; EUGENIA, 2019; MEZA, 2019) pero también desde las ciencias naturales y la educación ambiental, (METAUTE; FERNANDO, 2019; PÉREZ; ANAYA, 2017).

Desde los años 80 y 90 del Siglo pasado se ha buscado transformar la educación científica reflexionando sobre el aprendizaje con la idea constructivista piagetana como un proceso y la enseñanza como el ambiente que lo favorece (PEDRAZA-JIMÉNEZ, 2011). De esta manera, son diversos los enfoques de trabajo, sin embargo, las mismos 
Edição Especial: I SSAPEC - Simpósio Sul-Americano de Pesquisa em Ensino de Ciências

ISSN: 2595- $4520 \quad$ Vol. 4, n. 3. 2021

coinciden en que se deben incluir espacios de argumentación en los que se entienda como objetivos didácticos; la participación de los estudiantes desde un papel protagónico, con estrategias que propendan por el razonamiento, la justificación de sus puntos de vista, la solución de diferentes tipos de problemas de manera que se genere una postura crítica frente a la construcción del conocimiento y la utilidad que se le da.

En consecuencia, los conceptos que involucran las estrategias didácticas para el fortalecimiento de la argumentación son variados, sin embargo, se podría plantear que una de las necesidades mas latentes es la de contextualizar dichas estrategias en ejes de análisis de discusión, desde una visión multidisciplinar y multidimensional, que le permitan al estudiante reconocer la importancia del análisis de la problemática y la construcción cognitiva que sobre la misma se hace durante el espacio de clase, de manera que se pueda trascender desde este hacia el entorno real en el que el niño se desenvuelve.

Además, en el contexto epistemológico de la experiencia de aula, si bien la argumentación ofrece todas esas potencialidades mencionadas, es necesario resaltar que la argumentación en el aula, siguiendo a (RUIZ ORTEGA; TAMAYO ALZATE; MÁRQUEZ BARGALLÓ, 2015) es un proceso dialógico, en el que prima, el debate, la crítica, la toma de decisiones, la escucha y el respeto por el saber propio y del otro, por lo que debe suscitar en los estudiantes una justificación comprensible de las relaciones existentes entre, los datos, la información, el conocimiento y las afirmaciones, promoviendo así la capacidad de establecer criterios que permitan evaluar las intervenciones.

Un aporte importante, es el modelo argumentativo postulado por Toulmin que busca detectar los elementos o propiedades que describen o cualifican un buen argumento. Estos, acorde con su desarrollo conceptual y acudiendo al análisis que plantea Rodríguez-Bello (2004) de la obra de Toulmin, son: la tesis, la evidencia, las garantías que justifican, el respaldo que garantiza la certeza de las justificaciones, las garantías que dan importancia a las leyes, principios y patrones, el respaldo, la reserva y el cualificador.

En función a lo anterior es necesario que el desarrollo didáctico de la clase, convoque una serie de actores, estrategias y condiciones que provoquen tal confrontación, que propendan por el análisis y las herramientas sugeridas anteriormente, 
Edição Especial: I SSAPEC - Simpósio Sul-Americano de Pesquisa em Ensino de Ciências ISSN: 2595- $4520 \quad$ Vol. 4, n. 3. 2021

por lo que esta investigación acude a las problemáticas ambientales vistas desde su potencial multidimensional e interdisciplinario, y su relación de interdependencia con los problemas sociales (LIMA, 2009) posibilitando una interpretación de los retos y dificultades totalmente contextualizada que incorpore varias visiones de como las sociedades humanas han provocado esas dificultades y desde que puntos de solución se han empezado a tratar, uno de ellos la educación ambiental critica, la cual propendería por la búsqueda de medidas necesarias para preservar el planeta, sin la presión de procesos instrumentales, sino desde la construcción de comprensiones, concepciones y visiones de ideales de relación de los seres humanos con otras partes del sistema natural, como los recursos minerales, flora y de fauna. (WILDEMEERSCH, 2018)

En ese orden de ideas se visualiza una relación entre el análisis de la situación ambiental y la comprensión de los conceptos científicos sobre los cuales ya se han planteado relaciones en la enseñanza de las ciencias (MANCIPE, 2012; MERCHÁN, 2013; POSADA; GARCÍA, 2016), sin embargo, no se reconocen muchas propuestas didácticas que evalúen el potencial que esa relación plantea para el desarrollo de la argumentación en los sujetos participantes.

En función de los aportes educativos que se pueden generar desde la enseñanza de las ciencias se hace necesario generar espacios que permitan la confrontación de saberes, posturas, percepciones y que los mismos estén mediados por la realidad contextual del estudiante, pero también por los conocimientos científicos que el mismo incorpora o que puede apropiar a partir del compartir con sus compañeros y con la dirección y contribución de su docente a partir de lo cual, surge la pregunta de investigación ¿Cómo contribuye la articulación de los conocimientos científicos y el análisis de las problemáticas ambientales en el desarrollo de posturas argumentadas y fundamentadas en los niños de $5^{\circ}$ de educación primaria?

\section{METODOLOGÍA}

Esta investigación se desarrolló desde el paradigma cualitativo; que como lo plantea (SAMPIERI, 2018) parte de la observación de un fenómeno, que permite construir conocimiento previo sobre el análisis de narraciones, descripciones y otros insumos de colecta de información. Además se soporta en la Investigación Acción (IA) y en un planteamiento pedagógico constructivista (ALTAMIRANO; SALINAS, 2016). Recebido em: 18/01/2021 
Desde estas perspectivas se proyecta una secuencia didáctica desarrollada en siete (7) sesiones de clase, que involucra algunas herramientas conceptuales articuladas con problemáticas ambientales, que surgen del protagonismo de los niños, sus intereses y sus contexto experienciales cercanos, las mismas como se observa en la tabla 1, se plantean con un desarrollo didáctico que involucra entre otras cosas, la lectura de noticias que tienen que ver con el ambiente, columnas de opinión, estudios de caso específicos del contexto, imágenes, elementos multimedia, diseños creativos y todo mediado por un ambiente de discusión critica entorno a la situación particular que presenta cada recurso.

Tabla 1 Aspectos generales de las sesiones de clase, en función a los conceptos trabajados, las herramientas didácticas y las problemáticas ambientales involucradas.

\begin{tabular}{|c|c|c|c|}
\hline Sesión & Conceptos & Herramientas & Problemática \\
\hline 1 & $\begin{array}{l}\text { Hábitat } \\
\text { Ecosistemas } \\
\text { acuáticos }\end{array}$ & $\begin{array}{l}\text { Identificación de la } \\
\text { biodiversidad en el } \\
\text { país. } \\
\text { Estudio de caso } \\
\text { Consulta autónoma }\end{array}$ & $\begin{array}{l}\text { Humedales de Tunja el rescate } \\
\text { de la memoria de nuestros } \\
\text { padres. }\end{array}$ \\
\hline 2 & Peces & $\begin{array}{l}\text { Estudio de caso } \\
\text { Origami }\end{array}$ & $\begin{array}{l}\text { Ecoturismo el caso del buceo } \\
\text { con tiburones. }\end{array}$ \\
\hline evis & $\begin{array}{l}\text { Anfibios, ciclo } \\
\text { biológico de la rana. } \\
\text { a lnsionare }\end{array}$ & $\begin{array}{l}\text { Lectura de noticia } \\
\text { ambiental. } \\
\text { Origami. }\end{array}$ & $\begin{array}{l}\text { Problemáticas en anfibios, } \\
\text { intervención antrópica en } \\
\text { humedales. }\end{array}$ \\
\hline 4 & Anfibios & Discusión y debate & $\begin{array}{l}\text { Coctel venenoso, la rana } \\
\text { dorada del Chocó }\end{array}$ \\
\hline 5 & Reptiles & $\begin{array}{l}\text { Imágenes y elección } \\
\text { de problemática, } \\
\text { Origami. }\end{array}$ & $\begin{array}{l}\text { Comercialización de especies: } \\
\text { la piel de cocodrilo, llaveros } \\
\text { con tortugas, las mascotas } \\
\text { exóticas. }\end{array}$ \\
\hline 6 & Realimentación & $\begin{array}{l}\text { Película las aventuras } \\
\text { de Sammy } 1 .\end{array}$ & $\begin{array}{lr}\text { Calentamiento } & \text { global, } \\
\text { contaminación del } & \text { agua, } \\
\text { tráfico de especies. } & \end{array}$ \\
\hline 7 & Realimentación & $\begin{array}{l}\text { Consulta y análisis de } \\
\text { información. }\end{array}$ & $\begin{array}{ll}\text { Problemática } & \text { libre } \\
\text { investigación } & \end{array}$ \\
\hline
\end{tabular}


Es por eso que en cada sesión se manejó un detonante, que tiene que ver con las problemáticas ambientales, luego se inició un proceso de cuestionamiento, en el que se incita a los niños a pensar desde diferentes puntos de vista y modos de abordaje dicha problemática, recordando que lo que se busca es una visión multidimensional, integra e interdisciplinar de la misma, en ese proceso de cuestionamiento surge la necesidad en los estudiantes, de acudir a otras fuentes de información y lógicamente a los principios conceptuales e incluso epistemológico de las ciencias, de manera que puedan dar una interpretación de la problemática y generar una postura sobre la misma, a esta etapa se le denomino fundamentación.

Finalmente se pone en discusión cada una de las posturas y argumentos construidos en procesos individuales y colectivos entorno a las situaciones planteadas, a la vez que se empezaron a buscar puntos de convergencia y divergencia, sobre los cuales se plantean unas conclusiones, desde lo conceptual, lo procedimental y el rol que, como seres sociales, éticos y políticos tenemos en la solución o prevención de dicha situación problemática.

En ocasiones ante la escaza variabilidad de las posturas de los estudiantes, los docentes asumían el rol de villanos ambientales, en el que defendían principios económicos, decisiones políticas o acciones culturales que desequilibraban en el entorno natural y por ende generaban la problemática ambiental, esto con el fin de que los estudiantes lograran suficiente apropiación en la construcción argumentativa, y proyectaran cómo enfrentar realidades, que a diario se reflejan en las sociedades latinoamericanas.

El estudio se desarrolló con un grupo de quince (15) estudiantes del grado $5^{\circ}$ con edades entre los 11 y 12 años en el escenario de clases del pensamiento biológico ambiental, se utilizaron además las grabaciones de la clase y la compilación de las evidencias escritas de los estudiantes, que fueron colectadas a lo largo de cuatro (4) meses de intervención y sobre las cuales se fundamentaron los niveles de análisis de la Tabla 2.

Tabla 2 clasificación de los niveles de argumentación, adaptación de (JIMÉNEZ-ALEIXANDRE; ERDURAN, 2007)

\begin{tabular}{|c|c|}
\hline Nivel & Características \\
\hline Nivel 1 & Comprende los argumentos que son una descripción simple de la vivencia. \\
\hline
\end{tabular}


Edição Especial: I SSAPEC - Simpósio Sul-Americano de Pesquisa em Ensino de Ciências

ISSN: $2595-4520$

Vol. 4, n. 3. 2021

\begin{tabular}{|c|c|}
\hline Nivel 2 & $\begin{array}{c}\text { Comprende argumentos en los que se identifican con claridad los datos y } \\
\text { una conclusión. }\end{array}$ \\
\hline Nivel 3 & $\begin{array}{c}\text { Comprenden argumentos en los cuales se identifican con claridad los datos, } \\
\text { conclusiones y justificación. }\end{array}$ \\
\hline Nivel 4 & $\begin{array}{c}\text { Comprende argumentos constituidos por datos, conclusiones y } \\
\text { justificaciones, haciendo uso de cualificadores o respaldo teórico }\end{array}$ \\
\hline
\end{tabular}

\section{RESULTADOS}

Se lograron trabajar las 7 sesiones con 15 estudiantes, en las cuales se tuvo un avance en los niveles de argumentación, como se puede ver en la Tabla 3., se pasó de un nivel de argumentación de $0,73 \%$ en el grupo, el cual de acuerdo con (JIMÉNEZALEIXANDRE; ERDURAN, 2007), pertenece a comprender los argumentos que son una descripción simple de la vivencia, para después de la intervención evidenciar un nivel 2 en el que se comprenden argumentos en los que se identifican con claridad los datos y una conclusión.

Tabla 3: Resultados individuales de los estudiantes, de acuerdo al análisis que se realizó en cada una de las siete sesiones.

\begin{tabular}{|c|c|c|c|c|c|c|c|c|}
\hline Estudiantes & Sesión & Sesión & Sesión & Sesión & Sesión & Sesión & Sesión & Promedio \\
por & \\
& 1 & 2 & 3 & 4 & 5 & 6 & 7 & estudiante \\
\hline 1 & 0 & 0 & 0 & 0 & 0 & 0 & 0 & 0 \\
\hline 2 & 0 & 0 & 0 & 1 & 1 & 1 & 1 & 0.6 \\
\hline 3 & 1 & 1 & 1 & 3 & 3 & 3 & 4 & 2.3 \\
\hline 4 & 0 & 1 & 1 & 2 & 2 & 2 & 2 & 1.4 \\
\hline 5 & 1 & 2 & 2 & 2 & 2 & 1 & 3 & 1.9 \\
\hline 6 & 0 & 0 & 0 & 1 & 1 & 1 & 2 & 0.7 \\
\hline 7 & 1 & 1 & 0 & 2 & 2 & 1 & 1 & 1.1 \\
\hline 8 & 0 & 0 & 1 & 2 & 1 & 2 & 2 & 1.1 \\
\hline 9 & 3 & 3 & 3 & 4 & 3 & 2 & 4 & 3.1 \\
\hline 10 & 0 & 0 & 0 & 0 & 0 & 1 & 0 & 0.1 \\
\hline 11 & 0 & 0 & 0 & 1 & 1 & 2 & 3 & 1.0 \\
\hline 12 & 1 & 1 & 2 & 2 & 1 & 2 & 2 & 1.6 \\
\hline 13 & 2 & 2 & 0 & 0 & 1 & 2 & 2 & 1.3 \\
\hline
\end{tabular}




\begin{tabular}{|c|c|c|c|c|c|c|c|c|}
\hline 14 & 1 & 1 & 2 & 3 & 4 & 3 & 3 & 2.4 \\
\hline 15 & 1 & 1 & 1 & 0 & 0 & 1 & 1 & 0.7 \\
\hline $\begin{array}{c}\text { Promedio } \\
\text { grupal } \\
\text { por sesión }\end{array}$ & 0.73 & 0.87 & 0.87 & 1.53 & 1.47 & 1.60 & 2.00 & 1.30 \\
\hline
\end{tabular}

Se puede apreciar que los cambios son significativos (Tabla 3.) a nivel grupal, de igual forma que los procesos individuales están marcados por el interés y la motivación que cada sesión de clase género en los estudiantes, lo que constituye un aporte significativo en términos de las actitudes que se plantearon inicialmente (VAZQUEZ; MANASSERO MAS, 2008), pues lleva a comprender que las actitudes dependen del interés y motivación que generen las herramientas didácticas, analizados previamente en otros niveles de educación (ALEMÁN MARICHAL et al., 2018) y cuyas conclusiones se podrían complementar con mayor arraigo en el nivel de educación primaria.

Sin embargo los resultados también plantean que los estudiantes requieren un componente de aplicabilidad de los aprendizajes científicos que están adquiriendo y el asumir dentro de sus dinámicas lúdicas, roles de mayor protagonismo en la toma de decisiones, es decir unos escenarios que les permitan defender sus posturas y sentirse útiles, en la solución de las problemáticas que aquejan sus regiones, territorios y la vida en el planeta, pues se ve que en estas clases se presenta no solo el mayor dinamismo, sino un mayor fortalecimiento de las competencias argumentativas que se pretendían desarrollar con esta experiencia de aula.

Una exigencia una realidad que la pandemia plantea es el cambio en los paradigmas educativos, que implica el desarrollo didáctico del proceso educativo desde un enfoque vinculante, critico, participativo y motivante para el estudiante, aspectos que como se demuestra solo se logran desde los intereses y realidades de los mismos; así los contenidos tematicos pasen de ser un fin de la educación, para convertirse en el medio con el que se desarrollan actividades sociales responsables, se ejerce una democracia y participación real, o se proponen ejes de desarrollo cultural, social, científico y tecnológico para las regiones.

Lo anterior se concilia fácilmente con los postulados constructivistas que plantean que cada estudiante, tiene unos, saberes, métodos de estudio y estrategias con las que 
Edição Especial: I SSAPEC - Simpósio Sul-Americano de Pesquisa em Ensino de Ciências

ISSN: 2595- $4520 \quad$ Vol. 4, n. 3. 2021

adquiere un mayor desempeño, es decir se propende por un ser autónomo de su proceso (ALTAMIRANO; SALINAS, 2016) lo que se evidenció en la variabilidad al evaluar el desarrollo argumentativo de los estudiantes. El protagonismo del estudiante tiene como punto de partida las características de la subjetividad al individuo, proporcionándole una identidad propia y por lo tanto unas visiones entorno a su rol social, el desarrollo de sus habilidades y su propio proceso de construcción cognitiva e integral, que es independiente al método e intencionalidad de la educación, mas vincula una serie de factores pedagógicos, como los proporcionados por estrategias que tienen que ver con habilidades inherentes a cada uno de los proceso de su vida, en este caso, la habilidad de comunicarse de forma argumentada.

En la Tabla 4., se muestra el potencial de los resultados en términos de los cambios en el nivel de argumentación que tienen los estudiantes, comparando la sesión 1 con la sesión 7 de intervención, se evidenció que de 6 estudiantes ubicados inicialmente en el nivel 1, en la última sesión 3 de ellos avanzan a otros niveles superiores; 5 estudiantes lograron pasar a nivel 2, 3 pasaron a nivel 3 y 2 a nivel 4 ( esto nos ayuda a comprender el potencial argumentativo que tiene la aplicación conceptual de las ciencias naturales, aplicadas en el análisis de las problemáticas ambientales. Lastimosamente como se aprecia en la tabla 3, dos (2) estudiantes presentaron dificultades técnicas no tuvieron muy buenos resultados en cuanto no se recibieron insumos por parte de los mismos, que permitieran analizar su desempeño durante la aplicación de la estrategia. Esto refleja también la proporcionalidad de participación de la pandemia de 15 estudiantes 13 estuvieron activos y 2 presentaron dificultades técnicas durante el tiempo de aplicación, ello requiere entonces un trabajo personalizado que incluye lógicamente actividades diferentes, pensadas para el trabajo autónomo y la discusión únicamente dada entre docente y estudiante.

Tabla 4: cambio en los niveles de argumentación entre la sesión 1 y la sesión 7 de la intervención

\begin{tabular}{|c|c|c|}
\hline Nivel & Sesión 1 & Sesión 7 \\
\hline Nivel 1 & 6 estudiantes & 3 estudiantes \\
\hline Nivel 2 & 1 estudiante & 5 estudiantes \\
\hline Nivel 3 & 1 estudiante & 3 estudiantes \\
\hline Nivel 4 & 0 estudiantes & 2 estudiantes \\
\hline
\end{tabular}


la importancia de estos resultados se debe leer desde los retos presentados por la educación en tiempos de pandemia, el desarrollo de clases mediadas por la virtualidad y como de alguna forma el convertir las problemáticas en objeto de análisis y de propuesta argumentada frente a la solución de las mismas, garantiza un nuevo tipo de ciudadanía que desde sus primeros proceso educativos se acerca a su realidad, para comprenderla, desde las dinámicas conceptuales de las ciencias naturales, pero también desde las decisiones ciudadanas que desencadenan desequilibrios como el que actualmente afronta la humanidad o equilibrios en el ambiente que únicamente pueden surgir desde la toma de responsabilidades frente a las interacciones propias, con el otro y con lo otro.

\section{CONCLUSIONES}

Las problemáticas ambientales vinculadas con el desarrollo conceptual de las ciencias naturales se constituyen en pretextos pedagógicos y didácticos que potencializa la argumentación en la educación primaria, generando en los niños un mayor protagonismo en la realidad en la que se desenvuelven, que los lleva a pensarse su rol en el equilibrio ambiental, la utilidad del conocimiento científico en la solución de esas problemáticas, la importancia de argumentar y fundamentar sus posturas, para un ejercicio óptimo de su ciudadanía desde una visión ética y responsable, que prioriza el minimizar el impacto y daño ambiental causado por las actividades humanas y las relaciones con el entorno natural, lo que podría llevar a pensar este tipo de estrategias como un punto de partida de la educación ambiental en primaria.

Se desarrollaron y potenciaron escenarios de argumentación entorno a problemáticas ambientales que llevaron a los niños a pasar de una simple comprensión de la problemática desde su experiencia, hasta lograr ofrecer algunos datos, conclusiones y justificaciones en torno a las posturas que asumen frente a la realidad ambiental. Dichas posturas planteadas para contextos propios y lejanos, de igual forma los niños lograron confrontar sus conceptos, posturas y argumentos, con las intervenciones y modos de pensar del otro, encontrando en los procesos dialógicos una oportunidad de autoconstrucción y aportes a la construcción del otro y de lo otro, lo que implicaría un ejercicio democrático, participativo y activo, que satisface uno de los retos que las brechas educativas en los contextos latinoamericanos han venido planteando.

\section{REFERENCIAS}


ACEVEDO, J. A. Proyecto ROSE: relevancia de la educación científica. Revista Eureka sobre enseñanza y divulgación de las ciencias., v. 2, n. 3, p. 440-447, 2005.

ALEMÁN MARICHAL, B. et al. La motivación en el contexto del proceso enseñanzaaprendizaje en carreras de las Ciencias Médicas. Revista Médica Electrónica, v. 40, n. 4, p. 1257-1270, ago. 2018.

ALMUNA, E. A. Progreso, desarrollo y sustentabilidad ambiental en la ciudad: las tensiones sociedad- naturaleza en una hermenéutica de los territorios. Revista Márgenes Espacio Arte y Sociedad, v. 10, n. 13, p. 32-39, 8 dez. 2013.

ALTAMIRANO, A. L. R.; SALINAS, Z. B. La práctica dicente-investigativa desde la tecnología educativa y el socioconstructivismo / The researching practice of learners based on educational technology and socio-constructivism. Ciencia Unemi, v. 9, n. 17, p. 118-1124, 7 jun. 2016.

ANDERETE SCHWAL, M. Las desigualdades educativas durante la pandemia en la educación primaria de Argentina. Revista Andina de Educación, v. 4, n. 1, p. 5-10, 26 nov. 2020.

ARIAS, M. A.; SAPONARA, D. T. La actividad metaverbal en la enseñanza de la argumentación oral en niños de tercer grado de básica primaria. Zona próxima: revista del Instituto de Estudios Superiores en Educación, n. 25, p. 49-69, 2016.

ARNAL-BAILERA, A.; GASCA, B. Actividades con el ajedrez para trabajar la argumentación y la resolución de problemas en matemáticas en Educación Primaria. Números. Revista de Didáctica de las Matemáticas, v. 99, p. 71-84, nov. 2018.

BARRIOS, G. R.; ADAM, M. R. Factores determinantes en la producción científica de los grupos de investigación en Colombia. Revista española de documentación científica, v. 39, n. 1, p. 4, 2016.

BOTERO, M. B.; TOBÓN, D. S. J.; RAMÍREZ, J. S. A. Formación sociopolítica en la clase de ciencias: discusiones sobre el uso de agroquímicos como un asunto sociocientífico. Educación científica y ciudadanía en el siglo XXI: actas del VIII Congreso Iberoamericano de Educación Científica y del II Congreso Internacional de Pedagogía, Didáctica y TIC aplicadas a la Educación (CIEDUC 2015), Vol. 1, 2015 (Volumen 1), ISBN 978-84-16133-65-9, págs. 307-320. Anais... In: EDUCACIÓN CIENTÍFICA Y CIUDADANÍA EN EL SIGLO XXI: ACTAS DEL VIII CONGRESO IBEROAMERICANO DE EDUCACIÓN CIENTÍFICA Y DEL II CONGRESO INTERNACIONAL DE PEDAGOGÍA, DIDÁCTICA Y TIC APLICADAS A LA EDUCACIÓN (CIEDUC 2015). Servicio de Publicaciones, 2015Disponível em: $<$ https://dialnet.unirioja.es/servlet/articulo?codigo=5786965>. Acesso em: 7 jan. 2021

CABRERA, L.; PÉREZ, C. N.; SANTANA, F. ¿Se incrementa la desigualdad de oportunidades educativas en la Enseñanza Primaria con el cierre escolar por el coronavirus? International Journal of Sociology of Education, p. 27, 20 jun. 2020.

CERVANTES-BARRAZA, J.; VALBUENA, S.; PATERNINA, Y. Argumentos de estudiantes de primaria en el contexto del álgebra temprana. Educación y Humanismo, v. 21, n. 37 , p. $120-138,19$ jul. 2019.

CIFUENTES, R.; EUGENIA, M. Secuencia didáctica para favorecer la argumentación oral y escrita en grado segundo. 2 jul. 2019. 
GONZÁLEZ, Á. et al. Teaching in the pandemic: reconceptualizing Chilean educators' professionalism now and for the future. Journal of Professional Capital and Community, v. 5, n. 3/4, p. 265-272, 1 jan. 2020.

HERNÁNDEZ, B. A. R.; SERNA, C. N. M.; REYNA, N. S. R. Enseñar a argumentar en la escuela primaria: una secuencia didáctica basada en el género textual. Aula de encuentro: Revista de investigación y comunicación de experiencias educativas, v. 22, n. 1, p. 163-193, 2020.

HERRERA, M. A. P. Currículo Transversal en la Contemporaneidad. Escenarios, v. 14, n. 1, p. 85-101, 2016.

JIMÉNEZ, P.; YAMILE, O. La interdisciplinariedad en un proceso de ambientalización curricular en la Universidad Pedagógica y Tecnológica de Colombia. 2020.

JIMÉNEZ-ALEIXANDRE, M. P.; ERDURAN, S. Argumentation in Science Education: An Overview. In: ERDURAN, S.; JIMÉNEZ-ALEIXANDRE, M. P. (Eds.).

Argumentation in Science Education: Perspectives from Classroom-Based Research. Science \& Technology Education Library. Dordrecht: Springer Netherlands, 2007. p. 3-27.

LIMA, G. F. DA C. Educação ambiental crítica: do socioambientalismo às sociedades sustentáveis. Educação e Pesquisa, v. 35, n. 1, p. 145-163, abr. 2009.

MANCIPE, M. ENFOQUE CTSA Y LLUVIA ÁCIDA: APRÓXIMACIÓN A LA QUÍMICA PARTIENDO DE UNA CUESTIÓN SOCIO-CIENTÍFICA. P.P.D.Q. Boletín, n. 49, 7 jul. 2012.

MARTÍNEZ PÉREZ, L. F.; VILLAMIZAR FÚQUENE, D. P. Unidades didácticas sobre cuestiones socio científicas: construcciones entre la escuela y la universidad. 2014.

MERCHÁN, N. Y. T. Las Cuestiones Sociocientíficas: Una Alternativa De Educación Para La Sostenibilidad. Revista Luna Azul, n. 32, p. 45-51, 2011.

MERCHÁN, N. Y. T. EL USO DEL GLIFOSATO UNA CUESTION SOCIOCIENTÍFICA COMO CONTRIBUCION AL CUESTIONAMIENTO DE LA INFORMACION. Bio-grafía, p. 903.911-903.911, 2013.

MEZA, P. Aproximaciones a la función social de la argumentación en la escuela rural. Una mirada sociocultural. RHS-Revista Humanismo y Sociedad, v. 7, p. 37-51, 25 jun. 2019.

PEDRAZA-JIMENEZ, Y. Habilidades científicas en un ambiente constructivista para el aprendizaje de la química general". Educación para el siglo XXI: aportes del Centro de Investigación y Formación en Educación (CIFE), 2007-2010. En: Colombia, ed: Alfaomega Ediciones Uniandes , v. , p.197 - 220 ,2011.

PEDRAZA-JIMENEZ, Y. La Interdisciplinariedad en un proceso de ambientalización curricular en la Universidad Pedagógica y Tecnológica de Colombia. Tesis Doctoral. Universidad Distrital "FJC", 2020.

POSADA, J. F. P.; GARCÍA, J. J. G. Una didáctica para el estudio del benceno en productos de consumo masivo: aprendizaje con participación ciudadana. Indagatio Didactica, v. 8, n. 1, p. 1247-1266, 1 jan. 2016. 
Edição Especial: I SSAPEC - Simpósio Sul-Americano de Pesquisa em Ensino de Ciências

PRESTI, A. P. L. Identidad y educación: dilema de la contemporaneidad. Educere: Revista Venezolana de Educación, n. 34, p. 421-426, 2006.

RODRÍGUEZ-BELLO, L. I.; 0000-0002-7319-8041; ORCID. El modelo argumentativo de Toulmin en la escritura de artículos de investigación educativa. Revista Digital Universitaria (1607 - 6079). Vol. 5, No.1 (2004), 31 jan. 2004.

RUIZ ORTEGA, F. J.; TAMAYO ALZATE, O. E.; MÁRQUEZ BARGALLÓ, C. La argumentación en clase de ciencias, un modelo para su enseñanza. Educação e Pesquisa, v. 41, n. 3, p. 629-646, set. 2015.

SAMPIERI, R. H. METODOLOGÍA DE LA INVESTIGACIÓN: LAS RUTAS CUANTITATIVA, CUALITATIVA Y MIXTA. [s.1.] McGraw Hill Mexico, 2018.

TARTAVULEA, C. V. et al. Online Teaching Practices and the Effectiveness of the Educational Process in the Wake of the COVID-19 Pandemic. www.amfiteatrueconomic.ro, v. 22, n. 55, p. 920, ago. 2020.

VAZQUEZ, A.; MANASSERO MAS, M. A. El declive de las actitudes hacia la ciencia de los estudiantes: un indicador inquietante para la educación científica. Revista Eureka sobre Enseñanza y Divulgación de las Ciencias, v. 8, 1 set. 2008.

WILDEMEERSCH, D. Silence - a matter of public concern: reconsidering critical environmental and sustainability education. Environmental Education Research, v. 24, n. 9, p. 1371-1382, 2 set. 2018. 